\title{
Automata on Guarded Strings and Applications
}

\author{
Dexter Kozen* \\ Cornell University
}

February 7, 2003

\begin{abstract}
Guarded strings are like ordinary strings over a finite alphabet $P$, except that atoms of the free Boolean algebra on a set of atomic tests $B$ alternate with the symbols of $P$. The regular sets of guarded strings play the same role in Kleene algebra with tests as the regular sets of ordinary strings do in Kleene algebra.

In this paper we develop the elementary theory of finite automata on guarded strings, a generalization of the theory of finite automata on ordinary strings. We give several basic constructions, including determinization, state minimization, and an analog of Kleene's theorem.

We then use these results to verify a conjecture on the complexity of a complete Gentzen-style sequent calculus for partial correctness. We also show that a basic result of the theory of Boolean decision diagrams (BDDs), namely that minimal ordered BDDs are unique, is a special case of the Myhill-Nerode theorem for a class of automata on guarded strings.
\end{abstract}

\section{Introduction}

Guarded strings were introduced in [4] as an abstract interpretation for program schemes. Guarded strings are like ordinary strings over a finite alphabet $P$, except that atoms of the free Boolean algebra on a set of atomic tests $B$ alternate with the symbols of $P$. The regular sets of guarded strings over $\mathrm{P}$ and $\mathrm{B}$ form a Kleene algebra with tests (KAT) and play the same role in KAT as the regular sets of ordinary strings do in Kleene algebra; specifically, they form the free KAT on generators P,B [8].

Guarded strings are useful in other contexts. In [10], we developed a complete Gentzenstyle sequent calculus $S$ for partial correctness. Guarded strings played a central role

\footnotetext{
${ }^{*}$ Computer Science Department, Cornell University, Ithaca, NY 14853-7501, USA. kozen@cs.cornell.edu
} 
in the completeness proof. We also conjectured that the decision problem for $\mathrm{S}$ was PSPACE-complete.

In this paper we verify that conjecture. The proof requires the development the elementary theory of finite automata on guarded strings, a generalization of the theory of finite automata on ordinary strings. We give several basic constructions, including determinization, state minimization, and an analog of Kleene's theorem. We also point out a connection to the complexity of BDDs (binary or Boolean decision diagrams), a well-studied structure in model checking. In particular, we observe that a basic result of the theory of BDDs, namely that minimal ordered BDDs are unique, is a special case of the Myhill-Nerode theorem for a class of deterministic automata on guarded strings.

\section{Kleene Algebra with Tests and Guarded Strings}

\subsection{Kleene Algebra with Tests}

A Kleene algebra $\left(K,+, \cdot{ }^{*}, 0,1\right)$ is an idempotent semiring under $+, \cdot, 0,1$ such that $p^{*} q$ is the $\leq$-least solution to $q+p x \leq x$ and $q p^{*}$ is the $\leq$-least solution to $q+x p \leq x$, where $\leq$ refers to the natural partial order $p \leq q \stackrel{\text { def }}{\Longleftrightarrow} p+q=q$.

A Kleene algebra with tests (KAT) [6] is a two-sorted structure $\left(K, B,+, \cdot,^{*},-, 0,1\right)$ such that

- $\left(K,+, \cdot{ }^{*}, 0,1\right)$ is a Kleene algebra,

- $\left(B,+, \cdot,-{ }^{-}, 0,1\right)$ is a Boolean algebra, and

- $(B,+, \cdot, 0,1)$ is a subalgebra of $(K,+, \cdot, 0,1)$.

The Boolean complementation operator - ${ }^{-}$is defined only on $B$. Syntactically, the language of KAT contains two sorts of terms:

tests $\quad b, c, d \ldots \quad b::=\langle$ atomic tests $\rangle|\mathbf{0}| \mathbf{1}|b+c| b c \mid \bar{b}$

programs $\quad p, q, r, \ldots \quad p::=\langle$ atomic actions $\rangle|b| p+q|p q| p^{*}$

Standard examples of Kleene algebras include the family of regular sets over a finite alphabet, the family of binary relations on a set, the family of sets of traces of a labeled transition system, and the family of $n \times n$ matrices over another Kleene algebra. Other more exotic interpretations include the min,+ algebra or tropical semiring used in shortest path algorithms and models consisting of convex polyhedra used in computational geometry. 
All these Kleene algebras can be extended naturally to Kleene algebras with tests, but for applications in program verification, the extension makes the most sense in trace and relation algebras. For example, for the Kleene algebra of binary relations on a set $X$, a natural choice for the tests would be the subsets of the identity relation on $X$.

KAT subsumes propositional Hoare logic; moreover, unlike Hoare logic, KAT is deductively complete for relationally valid propositional Hoare-style rules involving partial correctness assertions [7].

We refer the reader to $[6,7]$ for a more thorough introduction to Kleene algebra with tests.

\subsection{Guarded Strings}

The family of regular sets of strings over a finite alphabet $P$ is the free Kleene algebra on generators $\mathrm{P}$. The structure that plays the analogous role in KAT is the family of regular sets of guarded strings. These objects were first studied by Kaplan in 1969 [4] as an abstract interpretation for program schemes.

Let $\mathrm{B}=\left\{b_{1}, \ldots, b_{k}\right\}$ and $\mathrm{P}=\left\{p_{1}, \ldots, p_{m}\right\}$ be fixed finite sets of atomic tests and atomic actions, respectively, and let $\overline{\mathrm{B}} \stackrel{\text { def }}{=}\{\bar{b} \mid b \in \mathrm{B}\}$. Tests and programs over $\mathrm{P}, \mathrm{B}$ were defined in Section 2.1. The set of all tests over $B$ is denoted $\mathcal{B}$.

An atom of $\mathrm{B}$ is a program $c_{1} \cdots c_{k}$ such that $c_{i} \in\left\{b_{i}, \bar{b}_{i}\right\}, 1 \leq i \leq k$, representing a minimal nonzero element of the free Boolean algebra on B. We can think of an atom as a truth assignment to $B$. Atoms are not to be confused with atomic tests, which are just the elements of $\mathrm{B}$. We denote by $\mathcal{A}_{\mathrm{B}}$ the set of all atoms of $\mathrm{B}$ and use the symbols $\alpha, \beta, \ldots$ exclusively for atoms. For an atom $\alpha$ and a test $b$, we write $\alpha \leq b$ if $\alpha \rightarrow b$ is a propositional tautology. For every atom $\alpha$ and test $b$, either $\alpha \leq b$ or $\alpha \leq \bar{b}$. Every element of a finite Boolean algebra can be represented as a disjoint sum of atoms.

A guarded string is a sequence $x=\alpha_{0} q_{0} \alpha_{1} \cdots \alpha_{n-1} q_{n-1} \alpha_{n}$, where $n \geq 0$ and each $\alpha_{i} \in \mathcal{A}_{\mathrm{B}}$ and $q_{i} \in \mathrm{P}$. We define first $(x)=\alpha_{0}$ and $\operatorname{last}(x)=\alpha_{n}$. The set of all guarded strings over $\mathrm{P}, \mathrm{B}$ is denoted GS. If $\operatorname{last}(x)=\boldsymbol{f i r s t}(y)$, we can form the fusion product $x y$ by concatenating $x$ and $y$, omitting the extra copy of the common atom. For example, if $x=\alpha p \beta$ and $y=\beta q \gamma$, then $x y=\alpha p \beta q \gamma$. If $\operatorname{last}(x) \neq \operatorname{first}(y)$, then $x y$ does not exist.

For sets $X, Y$ of guarded strings, define $X \circ Y$ to be the set of all existing fusion products $x y$ with $x \in X$ and $y \in Y$, and define $X^{n}$ to be the product of $n$ copies of $X$ with respect to this operation. Each program $p$ of KAT denotes a set $G(p)$ of guarded 
strings as follows:

$$
\begin{array}{rll}
G(p) & \stackrel{\text { def }}{=}\left\{\alpha p \beta \mid \alpha, \beta \in \mathcal{A}_{\mathrm{B}}\right\} \quad p \text { an atomic action } \\
G(b) & \stackrel{\text { def }}{=}\left\{\alpha \in \mathcal{A}_{\mathrm{B}} \mid \alpha \leq b\right\} & b \text { a test } \\
G(p+q) & \stackrel{\text { def }}{=} G(p) \cup G(q) & \\
G(p q) & \stackrel{\text { def }}{=} G(p) \circ G(q) & \\
G\left(p^{*}\right) & \stackrel{\text { def }}{=} & \bigcup \\
& & G(p)^{n} .
\end{array}
$$

A set of guarded strings over P,B is regular if it is $G(p)$ for some program $p$. The family of regular sets of guarded strings over $\mathrm{P}, \mathrm{B}$ is denoted $\operatorname{Reg}_{\mathrm{P}, \mathrm{B}}$. It forms the free Kleene algebra with tests on generators P,B [8]; in other words, $G(p)=G(q)$ iff $p=q$ is a theorem of KAT. A guarded string $x$ is itself a program, and $G(x)=\{x\}$. These are the minimal nonzero elements of $\operatorname{Reg}_{\mathrm{P}, \mathrm{B}}$.

A key lemma used in the completeness proof of [8] is the following result, essentially due to Kaplan [4]: for any program $p$, there exists a program $\widehat{p}$ such that $p=\widehat{p}$ is a theorem of KAT and $G(\widehat{p})=R(\widehat{p})$, where $R$ is the classical interpretation of regular expressions over the alphabet $\mathrm{P} \cup \mathrm{B} \cup \overline{\mathrm{B}}$ as regular subsets of $(\mathrm{P} \cup \mathrm{B} \cup \overline{\mathrm{B}})^{*}$. Moreover, if $R(q) \subseteq \mathbf{G S}$, then $R(q)=G(q)$; this is because $G(q)=\bigcup\{G(x) \mid x \in R(q)\}$ and $G(x)=\{x\}$ for $x \in$ GS. As observed in [10], this result implies that $\operatorname{Reg}_{\mathrm{P}, \mathrm{B}}$ is closed under the Boolean operations. Our automata-theoretic characterization of $\operatorname{Reg}_{\mathrm{P}, \mathrm{B}}$ will give an alternative proof of this result.

Programs of KAT can also be interpreted as sets of traces or sets of binary relations in Kripke frames. A Kripke frame over P,B is a structure $\left(K, \mathfrak{m}_{K}\right)$, where $K$ is a set of states and

$$
\mathfrak{m}_{K}: \mathrm{P} \rightarrow 2^{K \times K} \quad \mathfrak{m}_{K}: \mathrm{B} \rightarrow 2^{K} .
$$

A trace in $K$ is a sequence $\sigma$ of the form $s_{0} q_{0} s_{1} \cdots s_{n-1} q_{n-1} s_{n}$, where $n \geq 0$, $s_{i} \in K, q_{i} \in \mathrm{P}$, and $\left(s_{i}, s_{i+1}\right) \in \mathfrak{m}_{K}\left(q_{i}\right)$ for $0 \leq i \leq n-1$. The length of $s_{0} q_{0} s_{1} \cdots s_{n-1} q_{n-1} s_{n}$ is $n$. We define $\operatorname{first}(\sigma)=s_{0}, \operatorname{last}(\sigma)=s_{n}$, and label $(\sigma)=$ $q_{0} \cdots q_{n-1}$. For traces of length $0, \operatorname{label}(s)=1$. If last $(\sigma)=\operatorname{first}(\tau)$, the trace $\sigma \tau$ is the trace consisting of $\sigma$ followed by $\tau$. If $\operatorname{last}(\sigma) \neq \operatorname{first}(\tau)$, then $\sigma \tau$ does not exist. A trace $s_{0} q_{0} s_{1} \cdots s_{n-1} q_{n-1} s_{n}$ is linear if the $s_{i}$ are distinct.

Programs are interpreted in $K$ as sets of traces according to the following inductive 
definition:

$$
\begin{aligned}
& \llbracket p \rrbracket_{K} \stackrel{\text { def }}{=}\left\{s p t \mid(s, t) \in \mathfrak{m}_{K}(p)\right\}, \quad p \text { an atomic action } \\
& \llbracket b \rrbracket_{K} \stackrel{\text { def }}{=} \mathfrak{m}_{K}(b), \quad b \text { an atomic test } \\
& \llbracket[\bar{b}]_{K} \stackrel{\text { def }}{=} K-\mathfrak{m}_{K}(b) \\
& \llbracket 0 \rrbracket_{K} \stackrel{\text { def }}{=} \varnothing \\
& \left.\llbracket p+q]_{K} \stackrel{\text { def }}{=} \llbracket[p]_{K} \cup \llbracket q\right]_{K} \\
& \left.\llbracket p q \rrbracket_{K} \stackrel{\text { def }}{=} \llbracket[p]_{K} \circ \llbracket q\right]_{K} \\
& \llbracket p^{*} \rrbracket_{K} \stackrel{\text { def }}{=} \bigcup_{n \geq 0} \llbracket p \rrbracket_{K}^{n},
\end{aligned}
$$

where $X \circ Y \stackrel{\text { def }}{=}\{\sigma \tau \mid \sigma \in X, \tau \in Y, \sigma \tau$ exists $\}$ and $X^{0} \stackrel{\text { def }}{=} K, X^{n+1} \stackrel{\text { def }}{=} X \circ X^{n}$.

Every trace $\sigma$ has an associated guarded string gs $(\sigma)$ defined by

$$
\operatorname{gs}\left(s_{0} q_{0} s_{1} \cdots s_{n-1} q_{n-1} s_{n}\right) \stackrel{\text { def }}{=} \alpha_{0} q_{0} \alpha_{1} \cdots \alpha_{n-1} q_{n-1} \alpha_{n},
$$

where $\alpha_{i}$ is the unique atom of B such that $s_{i} \in \llbracket \alpha_{i} \rrbracket_{K}$, and $\operatorname{gs}(\sigma)$ is the unique guarded string over $\mathrm{P}, \mathrm{B}$ such that $\sigma \in[\operatorname{gs}(\sigma)]_{K}$. The relationship between trace semantics and guarded strings is given by the following lemma.

Lemma 2.1 ([10]) In any trace model $K$, for any program $p$ and trace $\tau, \tau \in \llbracket p]_{K}$ iff $\operatorname{gs}(\tau) \in G(p)$. In other words, $\llbracket p \rrbracket_{K}=\mathrm{gs}^{-1}(G(p))$. The map $X \mapsto \mathrm{gs}^{-1}(X)$ is a KAT homomorphism from the algebra of regular sets of guarded strings to the algebra of regular sets of traces over $K$.

\section{Automata on Guarded Strings}

A finite automaton on guarded strings (AGS) over atomic actions $\mathrm{P}$ and atomic tests $B$ is just an ordinary finite automaton with transition labels $\mathrm{P} \cup \mathcal{B}$, where $\mathcal{B}$ is the set of tests built from atomic tests $B$, except that acceptance is defined differently. Strictly speaking, $\mathcal{B}$ is infinite; however, it is finite up to propositional equivalence, and the semantics of acceptance does not distinguish propositionally equivalent tests. Transitions labeled with atomic actions are called action transitions and those labeled with tests are called test transitions.

Ordinary finite automata with $\varepsilon$-transitions can be regarded as the special case in which $\mathrm{B}=\varnothing$, giving the two-element Boolean algebra $\{\mathbf{0}, \mathbf{1}\}$. An $\varepsilon$-transition is just a test transition with Boolean label 1. For nonempty B, tests can be more complicated.

Intuitively, nondeterministic automata on guarded strings work as follows. An input to the automaton is a guarded string over $\mathrm{P}$ and $\mathrm{B}$. We start with a pebble on an input 
state with the input pointer reading the first atom of the input string. At any point in the computation, the pebble is occupying a state, and the input pointer is pointing to an atom somewhere in the input string. If there is an action transition from the current state labeled with $p \in \mathrm{P}$, and the next program symbol in the input string is $p$, then we may nondeterministically choose to move the pebble along that transition and advance the input pointer beyond $p$. If there is a test transition from the current state labeled with a test $b \in \mathcal{B}$, and if that transition is enabled, then we may nondeterministically choose to move the pebble along that transition, but we do not advance the input pointer. The transition is enabled if the current atom $\alpha$ in the input string satisfies $b$, where we regard $\alpha$ as a truth assignment to $\mathrm{B}$. The input is accepted if the pebble occupies an accept state while the input pointer is pointing to the last atom in the input string.

Formally, an automaton on guarded strings over $\mathrm{P}, \mathrm{B}$ is a Kripke frame $M=\left(Q, \mathfrak{m}_{M}\right)$ over atomic actions $\mathrm{P} \cup \mathcal{B}$ and atomic tests $\varnothing$, along with a distinguished set $S \subseteq Q$ of start states and a distinguished set $F \subseteq Q$ of final or accept states. We write $u \underset{M}{\stackrel{d}{\longrightarrow}} v$ if $(u, v) \in \mathfrak{m}_{M}(d), d \in \mathrm{P} \cup \mathcal{B}$, or just $u \stackrel{d}{\longrightarrow} v$ if $M$ is understood.

A guarded string $y$ over $\mathrm{P}, \mathrm{B}$ is said to be accepted by $M$ if $y \in G(x)$ for some $x \in R(M)$, where $R(M)$ is the set of strings in $(\mathrm{P} \cup \mathcal{B})^{*}$ accepted by $M$ under the ordinary definition of finite automaton acceptance. The set of all guarded strings over P, B accepted by $M$ is denoted $G(M)$. Formally,

$$
\begin{aligned}
& R(M) \stackrel{\text { def }}{=}\{\operatorname{label}(\sigma) \mid \operatorname{first}(\sigma) \in S, \operatorname{last}(\sigma) \in F\} \\
& G(M) \stackrel{\text { def }}{=} H(R(M)),
\end{aligned}
$$

where $\sigma$ represents a trace in $M$ and $H$ is the map

$$
\begin{gathered}
H: 2^{(\mathrm{P} \cup \mathcal{B})^{*}} \rightarrow 2^{\mathrm{GS}} \\
H(A) \stackrel{\text { def }}{=} \bigcup_{x \in A} G(x) .
\end{gathered}
$$

\subsection{Kleene's Theorem}

The following is the analog of Kleene's theorem for automata on guarded strings. We need the second clause for our complexity result in Section 4.1.

Theorem 3.1 Automata on guarded strings over P, B accept all and only regular sets. Moreover, the size of the equivalent automaton $M$ constructed from a given program $p$ is linear in the size of $p$.

Proof. Given a program $p$ over P, B, consider it as a regular expression over the alphabet $\mathrm{P} \cup \mathcal{B}$ with the classical interpretation, and construct an equivalent finite automaton $M$ with input alphabet $\mathrm{P} \cup \mathcal{B}$ as in the usual proof of Kleene's theorem (see e.g. [5]). 
The construction is linear. Conversely, given a finite automaton $M$ with input alphabet $\mathrm{P} \cup \mathcal{B}$, construct an equivalent regular expression $p$. In either direction, let $R(p)$ denote the regular subset of $(\mathrm{P} \cup \mathcal{B})^{*}$ denoted by $p$ under the classical interpretation of regular expressions, and let $R(M)$ denote the subset of $(\mathrm{P} \cup \mathcal{B})^{*}$ accepted by $M$ under the classical semantics of finite automata. By Kleene's theorem, $R(p)=R(M)$.

We claim that in both constructions, $G(p)=G(M)$ as well. To show this, it suffices to show that

$$
\begin{aligned}
G(M) & =H(R(M)) \\
G(p) & =H(R(p)) .
\end{aligned}
$$

The equation (1) is just the definition of acceptance for automata on guarded strings. The equation (2) was proved in [2]. Briefly, it is easily shown that the map $H$ is a homomorphism with respect to the operators $\cup$, ०, and *. Moreover, the maps $G$ and $H \circ R$ agree on the generators $\mathrm{P}$ and $\mathcal{B}$, since $H(R(d))=H(\{d\})=G(d)$ for $d \in \mathrm{P} \cup \mathcal{B}$, and $H(R(\mathbf{0}))=G(\mathbf{0})=\varnothing$. It follows by induction that $G$ and $H \circ R$ agree on all regular expressions over $\mathrm{P} \cup \mathcal{B}$.

\subsection{Determinization}

In this section we show how to construct a deterministic automaton on guarded strings equivalent to a given nondeterministic one. This is the basis of our PSPACE algorithm of Section 4.1. The construction is analogous to the standard subset construction for automata on ordinary strings (see e.g. [5]).

An automaton $M$ on guarded strings is deterministic if it satisfies the following properties.

(i) There is exactly one start state.

(ii) Each state may have either exiting action transitions or exiting test transitions, but not both. A state is called an action state or a test state in these two circumstances, respectively. Every state is either an action state or a test state.

(iii) Every action state has exactly one exiting action transition for each element of P.

(iv) The labels of the exiting test transitions of a test state are pairwise exclusive and exhaustive. By this we mean that if the labels are $c_{1}, \ldots, c_{n}$, then $\bar{c}_{i}+\bar{c}_{j}$ for $i \neq j$ and $c_{1}+\cdots+c_{n}$ are propositional tautologies.

(v) Every cycle contains at least one action transition.

(vi) All final states are action states. 
Note that $M$ is not a deterministic automaton in the classical sense. Conditions (i) and (iii) are standard for deterministic automata. Condition (ii) ensures that there is no ambiguity in whether to continue to test Boolean inputs or whether to read the next atomic action. Condition (iv) ensures that at any test state, exactly one exiting transition is enabled. Condition (v) ensures that there can be no endless loop of tests. Condition (vi) forces all pending tests to be resolved before deciding whether to accept the input.

Lemma 3.2 For any $x \in \mathrm{GS}$ and state $u$ of a deterministic AGS $M$, there is a unique maximal trace $\sigma_{M}(u, x)$ of $M$ such that $\operatorname{first}\left(\sigma_{M}(u, x)\right)=u$ and $x \in G\left(\operatorname{label}\left(\sigma_{M}(u, x)\right)\right)$. Moreover, last $\left(\sigma_{M}(u, x)\right)$ is an action state.

Proof. This follows from the conditions of determinacy by induction on the length of $x$.

We can convert a given nondeterministic automaton $N$ to an equivalent deterministic automaton $M$ by a subset construction. Suppose $N$ has states $Q$, transition relation $\mathfrak{m}_{N} \subseteq Q \times(\mathrm{P} \cup \mathcal{B}) \times Q$, start states $S \subseteq Q$, and final states $F \subseteq Q$. Define $M$ with states $Q^{\prime}=2^{Q} \times\{\mathbf{a}, \mathbf{t}\}$ and deterministic transition relation $\mathfrak{m}_{M} \subseteq Q^{\prime} \times(\mathrm{P} \cup$ $\left.\mathcal{A}_{\mathrm{B}}\right) \times Q^{\prime}$ as follows. The tags $\mathbf{a}, \mathbf{t}$ determine whether the state is an action or a test state, respectively.

$$
\begin{aligned}
(U, \mathbf{a}) \stackrel{p}{M}(V, \mathbf{t}) \stackrel{\text { def }}{\Longleftrightarrow} V & =\{v \mid \exists u \in U u \stackrel{p}{N} v\} \\
& =\{\operatorname{last}(\tau) \mid \operatorname{first}(\tau) \in U, p=\operatorname{label}(\tau)\} \\
(U, \mathbf{t}) \stackrel{\alpha}{M}(V, \mathbf{a}) \stackrel{\operatorname{def}}{\Longleftrightarrow} V & =\{\operatorname{last}(\pi) \mid \operatorname{first}(\pi) \in U, \alpha \leq \operatorname{label}(\pi)\} \\
& =\{\operatorname{last}(\pi) \mid \operatorname{first}(\pi) \in U, \alpha \in G(\operatorname{label}(\pi))\},
\end{aligned}
$$

where $\tau$ and $\pi$ represent traces of $N, p \in \mathrm{P}$, and $\alpha \in \mathcal{A}_{\mathrm{B}}$. The unique start state of $M$ is $(S, \mathbf{t})$ and the final states are $\{(E, \mathbf{a}) \mid E \cap F \neq \varnothing\}$.

Thus $(U, \mathbf{a}) \underset{M}{\stackrel{p}{M}}(V, \mathbf{t})$ iff $V$ is the set of states of $N$ reachable from a state in $U$ via a single transition with label $p$, and $(U, \mathbf{t}) \underset{M}{\stackrel{\alpha}{\longrightarrow}}(V, \mathbf{a})$ iff $V$ is the set of states of $N$ reachable from a state in $U$ via a trace whose label is a sequence of tests, all of which are satisfied by $\alpha$. Figure 1 illustrates this construction for a nondeterministic automaton over $\mathrm{P}=\{p\}$ and $\mathrm{B}=\{b, c\}$. The set of guarded strings accepted by the two machines in Figure 1 is $\{b \bar{c}, \bar{b} \bar{c}\} \cup\left\{b c p \alpha, b \bar{c} p \alpha \mid \alpha \in \mathcal{A}_{\mathrm{B}}\right\}$.

The automaton $M$ constructed above is evidently deterministic. Property (v) follows from the fact that the graph of $M$ is bipartite between action and test states. Properties (iii) and (iv) follow from the fact that $V$ on the right-hand side of the definition of the transition relation is unique, and that the atoms of $B$ are pairwise exclusive and exhaustive. Properties (i), (ii), and (vi) are immediate from the construction. 

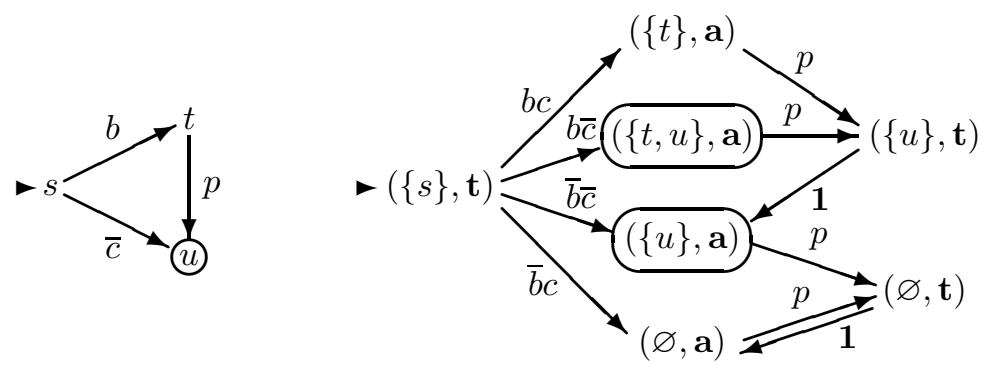

Figure 1: A nondeterministic automaton and an equivalent deterministic automaton

Since the graph of $M$ is bipartite between action and test states, and since all test labels are atoms, the label of any trace consists of alternating atomic actions and atoms of B. Thus if $\operatorname{first}(\tau)=(U, \mathbf{t})$ and $\operatorname{last}(\tau)=(V, \mathbf{a})$, then label $(\tau)$ begins and ends with an atom, so it is a guarded string. Since the start state is of the form $(S, \mathbf{t})$ and the final states are all of the form $(E, \mathbf{a})$, any string accepted by $M$ is a guarded string, therefore $R(M) \subseteq$ GS.

It follows from these remarks and Lemma 3.2 that for all $x \in \mathbf{G S}$ and $U \subseteq Q$, the unique maximal trace $\sigma_{M}((U, \mathbf{t}), x)$ of $M$ determined by $(U, \mathbf{t})$ and $x$ not only has $x \in G\left(\operatorname{label}\left(\sigma_{M}((U, \mathbf{t}), x)\right)\right)$, but actually $x=\operatorname{label}\left(\sigma_{M}((U, \mathbf{t}), x)\right)$. Moreover, last $\left.\sigma_{M}((U, \mathbf{t}), x)\right)$ is of the form $(V, \mathbf{a})$. Let us denote by $\Delta(U, x)$ the set $V$ uniquely determined by $U$ and $x$ in this way.

Lemma 3.3 For all $x \in \mathbf{G S}$ and $U \subseteq Q$,

$$
\Delta(U, x)=\{\operatorname{last}(\sigma) \mid \operatorname{first}(\sigma) \in U, x \in G(\operatorname{label}(\sigma))\},
$$

where $\sigma$ ranges over traces of $N$.

Proof. We proceed by induction on the length of $x$. The basis $x=\alpha \in \mathcal{A}_{\mathrm{B}}$ is just the definition of $\underset{M}{\stackrel{\alpha}{\longrightarrow}}$. For $x$ of the form $y p \alpha$, by the definition of $\underset{M}{\stackrel{\alpha}{\longrightarrow}}$ and $\underset{M}{\stackrel{p}{\longrightarrow}}$ and the 
induction hypothesis, we have

$$
\begin{aligned}
& \Delta(U, x) \\
& =\{\operatorname{last}(\pi) \mid \operatorname{first}(\pi) \in\{\operatorname{last}(\tau) \mid \operatorname{first}(\tau) \in \Delta(U, y), p=\operatorname{label}(\tau)\}, \\
& \alpha \in G(\operatorname{label}(\pi))\} \\
& =\{\operatorname{last}(\pi) \mid \boldsymbol{f i r s t}(\pi) \in\{\operatorname{last}(\tau) \mid \boldsymbol{f} \operatorname{frst}(\tau) \in\{\operatorname{last}(\sigma) \mid \operatorname{first}(\sigma) \in U, \\
& y \in G(\operatorname{label}(\sigma))\}, p=\operatorname{label}(\tau)\}, \alpha \in G(\operatorname{label}(\pi))\} \\
& =\{s \mid \exists \sigma \exists \tau \exists \pi s=\operatorname{last}(\pi), \operatorname{first}(\pi)=\operatorname{last}(\tau), \operatorname{first}(\tau)=\operatorname{last}(\sigma) \text {, } \\
& \operatorname{first}(\sigma) \in U, y \in G(\operatorname{label}(\sigma)), p=\operatorname{label}(\tau), \alpha \in G(\operatorname{label}(\pi))\} \\
& =\{s \mid \exists \xi \exists \sigma \exists \tau \exists \pi \xi=\sigma \tau \pi, s=\operatorname{last}(\xi), \operatorname{first}(\xi) \in U \text {, } \\
& y \in G(\operatorname{label}(\sigma)), p=\operatorname{label}(\tau), \alpha \in G(\operatorname{label}(\pi))\} \\
& =\{\operatorname{last}(\xi) \mid \boldsymbol{f i r s t}(\xi) \in U, \exists \sigma \exists \tau \exists \pi \xi=\sigma \tau \pi \text {, } \\
& y \in G(\operatorname{label}(\sigma)), p=\operatorname{label}(\tau), \alpha \in G(\operatorname{label}(\pi))\} \\
& =\{\operatorname{last}(\xi) \mid \operatorname{first}(\xi) \in U, \text { yp } \alpha \in G(\operatorname{label}(\xi))\} \\
& =\{\operatorname{last}(\xi) \mid \boldsymbol{f i r s t}(\xi) \in U, x \in G(\operatorname{label}(\xi))\} \text {. }
\end{aligned}
$$

Theorem $3.4 G(M)=G(N)$.

Proof. We have argued that $R(M) \subseteq$ GS. Since $G(x)=\{x\}$ for guarded strings $x$, $H$ is the identity on subsets of GS, therefore

$$
G(M)=H(R(M))=R(M)
$$

Now using Lemma 3.3,

$$
\begin{aligned}
R(M) & =\{x \mid \Delta(S, x) \cap F \neq \varnothing\} \\
& =\{x \mid\{\operatorname{last}(\sigma) \mid \operatorname{first}(\sigma) \in S, x \in G(\operatorname{label}(\sigma))\} \cap F \neq \varnothing\} \\
& =\{x \mid \exists \sigma \operatorname{first}(\sigma) \in S, x \in G(\operatorname{label}(\sigma)), \operatorname{last}(\sigma) \in F\} \\
& =G(N) .
\end{aligned}
$$

\subsection{State Minimization}

It turns out that the existence of unique minimal deterministic AGSs depends on the choice of input alphabet and restictions on how inputs can be read. We show in Section 3.3.2 that if any test in $\mathcal{B}$ is allowed as an input symbol, unique minimal deterministic AGSs exist. The test symbols of the minimal AGS can be taken to be the atoms of B. However, although the number of states is small, the specification of transitions may be exponential in the size of $B$. 
A more reasonable choice of input alphabet for tests is $B \cup \bar{B}$. There is no loss of generality in this restriction, since all regular sets of guarded strings can still be represented, but the number of states may increase. Unfortunately, uniqueness of minimal automata is no longer guaranteed. However, if the automata are constrained to read their Boolean inputs in a given fixed order — such automata are called ordered - then minimal automata are unique. We show in Section 4.2 that the Canonicity Lemma for reduced ordered Boolean decision diagrams (ROBDDs) (see [1]) is a special case of this result.

\subsubsection{Ordered AGSs}

If we restrict the input alphabet to $\mathrm{P} \cup \mathrm{B} \cup \overline{\mathrm{B}}$, uniqueness of minimal deterministic automata is not guaranteed. For example, the automata
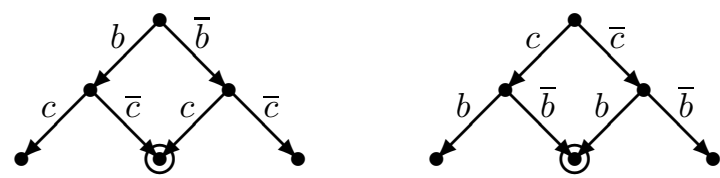

over $\mathrm{P}=\varnothing$ and $\mathrm{B}=\{b, c\}$ represent the same set of guarded strings $\{b \bar{c}, \bar{b} c\}$. However, uniqueness can be guaranteed provided we constrain the automata to test Boolean inputs in a particular fixed order, say $b_{1}, b_{2}, \ldots, b_{k}$. In such automata, each test state is assigned a level between 0 and $k-1$, inclusive. A test state at level $i$ has one exiting transition labeled $b_{i+1}$ and one labeled $\bar{b}_{i+1}$, and the transitions must go either to an action state or to a higher-level test state. Such an AGS is called ordered. We show that there is a unique minimal deterministic ordered AGS with respect to the given linear order on B. The construction is a generalization of the Myhill-Nerode construction of classical finite automata theory (see [5]).

Recall our definition of an atom of $\mathrm{B}=\left\{b_{1}, \ldots, b_{k}\right\}$ as a string $c_{1} \cdots c_{k}$ such that $c_{i} \in\left\{b_{i}, \bar{b}_{i}\right\}, 1 \leq i \leq k$. A prefix of an atom is a string of the form $c_{1} \cdots c_{j}$, where $0 \leq j \leq k$ and $c_{i} \in\left\{b_{i}, \bar{b}_{i}\right\}, 1 \leq i \leq j$. The empty string is allowed; that is the case $j=0$. The symbol $\omega$ is used for prefixes of atoms.

A prefix of a guarded string is a string $x$ over $\mathrm{P} \cup \mathrm{B} \cup \overline{\mathrm{B}}$ such that $x y \in \mathbf{G S}$ for some string $y$ over $P \cup B \cup \bar{B}$. The definition of prefix is with respect to the ordinary string concatenation operation on strings over $P \cup B \cup \bar{B}$. A prefix of a guarded string is similar to a guarded string, except that last $(x)$ may be a prefix of an atom. The set of all prefixes of guarded strings is denoted PGS.

We define a binary operation $\triangleleft$ on PGS as follows. If $c_{1} \cdots c_{m}$ and $d_{1} \cdots d_{n}$ are prefixes of atoms, define

$$
c_{1} \cdots c_{m} \triangleleft d_{1} \cdots d_{n} \stackrel{\text { def }}{=} \begin{cases}c_{1} \cdots c_{m}, & \text { if } n \leq m \\ c_{1} \cdots c_{m} d_{m+1} \cdots d_{n}, & \text { otherwise. }\end{cases}
$$


Intuitively, we overlay $c_{1} \cdots c_{m}$ on $d_{1} \cdots d_{n}$, resolving disagreements in favor of the $c_{i}$. If $m=0$, the result is just $d_{1} \cdots d_{n}$. For $x, y \in \mathbf{P G S}$, we define $x \triangleleft y$ similarly to the fusion product $x y$, except that we perform $\triangleleft$ at the interface instead of fusing $\operatorname{last}(x)$ and $\operatorname{first}(y)$ as in the fusion product. Unlike fusion product, $\triangleleft$ is a total operation. For example,

$$
\begin{aligned}
b \bar{c} d p \bar{b} c \triangleleft b c \bar{d} q \bar{b} \bar{c} & =b \bar{c} d p \bar{b} c \bar{d} q \bar{b} \bar{c} \\
b \bar{c} d p \bar{b} \bar{c} d \triangleleft b c & =b \bar{c} d p \bar{b} \bar{c} d .
\end{aligned}
$$

It is easily shown that $\triangleleft$ is associative.

Now let $A \subseteq$ GS. For $x, y \in \mathbf{P G S}$, define the Myhill-Nerode relation

$$
x \equiv y \stackrel{\text { def }}{\Longleftrightarrow} \forall z \in \mathbf{G S}(x \triangleleft z \in A \Leftrightarrow y \triangleleft z \in A) .
$$

Lemma 3.5 If $x, y \in \mathbf{P G S}$ and $x \equiv y$, then $x \triangleleft z \equiv y \triangleleft z$ for any $z \in \mathbf{P G S}$.

Proof. Using the associativity of $\triangleleft$, for any $w \in \mathbf{G S},(x \triangleleft z) \triangleleft w \in A$ iff $x \triangleleft(z \triangleleft$ $w) \in A$ iff $y \triangleleft(z \triangleleft w) \in A$ iff $(y \triangleleft z) \triangleleft w \in A$.

Lemma 3.6 If $x, y \in \mathbf{G S}$ and $x \equiv y$, then $x \in A$ iff $y \in A$.

Proof. For any atom $\alpha, x \in A$ iff $x \triangleleft \alpha \in A$ iff $y \triangleleft \alpha \in A$ iff $y \in A$.

$\operatorname{Define} \operatorname{level}(x)$ to be the maximum value of $|\operatorname{last}(y)|$ over all $y \equiv x$, where $|\omega|$ is the length of the atom prefix $\omega$ regarded as a string over $\mathrm{B} \cup \overline{\mathrm{B}}$. Then $0 \leq \operatorname{level}(x) \leq k$, and level $(x)=k$ iff $x$ is $\equiv$-equivalent to a guarded string.

We now build a deterministic ordered AGS $N$ over $\mathrm{P} \cup \mathrm{B} \cup \overline{\mathrm{B}}$ from the equivalence classes of $\equiv$. Let

$$
[x] \stackrel{\text { def }}{=}\{y \in \mathbf{P G S} \mid y \equiv x\} .
$$

The states of $N$ are $\{[x] \mid x \in \mathbf{P G S}\}$. A state $[x]$ is a test state if $0 \leq \operatorname{level}(x) \leq$ $k-1$ and an action state if $\operatorname{level}(x)=k$. The transitions are

$$
\begin{array}{ll}
\left.\begin{array}{ll}
{[x] \underset{N}{\stackrel{b_{i}}{\longrightarrow}}\left[x b_{i}\right]} \\
{[x] \underset{\bar{b}_{i}}{\stackrel{N}{\longrightarrow}}\left[x \bar{b}_{i}\right]}
\end{array}\right\} & \text { if level }(x)=|\operatorname{last}(x)|=i-1<k \\
{[x] \underset{N}{\stackrel{p}{\longrightarrow}}[x p]} & \text { if level }(x)=|\operatorname{last}(x)|=k .
\end{array}
$$

The start state of $N$ is $[\varepsilon]$ and the final states are $\{[x] \mid x \in A\}$. The transitions are well defined by Lemma 3.5, and $x \in A$ iff $[x]$ is a final state by Lemma 3.6. 
By Lemma 3.2, for any $x \in \mathbf{G S}$, there exists a unique maximal trace $\sigma_{N}([\varepsilon], x)$ such that first $\left(\sigma_{N}([\varepsilon], x)\right)=[\varepsilon]$ and $x \in G\left(\operatorname{label}\left(\sigma_{N}([\varepsilon], x)\right)\right)$. We will show in Lemma 3.10 that $\operatorname{last}\left(\sigma_{N}([\varepsilon], x)\right)=[x]$.

For any $x, y \in$ PGS, let $\sigma_{N}([x], y)$ be the longest common prefix of the traces $\sigma_{N}([x], y \triangleleft \alpha)$ for all atoms $\alpha$. We denote this by $\mathbf{l c p}_{\alpha} \sigma_{N}([x], y \triangleleft \alpha)$.

Lemma 3.7 Let $x \in \mathbf{P G S}$ and let $\omega$ be a prefix of an atom. If $x \triangleleft \eta \equiv x \triangleleft \omega$ for all $\eta$ such that $|\eta|=|\omega|$, then $x \equiv x \triangleleft \omega$.

Proof. For any $z \in \mathbf{G S}$, let $\eta_{z}$ be the prefix of $\operatorname{first}(z)$ of length $|\omega|$. Then

$$
\begin{aligned}
x \triangleleft z \in A & \Leftrightarrow x \triangleleft\left(\eta_{z} \triangleleft z\right) \in A \\
& \Leftrightarrow \quad\left(x \triangleleft \eta_{z}\right) \triangleleft z \in A \\
& \Leftrightarrow \quad(x \triangleleft \omega) \triangleleft z \in A .
\end{aligned}
$$

Since $z$ was arbitrary, $x \equiv x \triangleleft \omega$.

Lemma 3.8 The two successors of any test state in $N$ are distinct.

Proof. Let $[x]$ be a test state. Assume without loss of generality that $|\operatorname{last}(x)|=$ $\operatorname{level}([x])=i-1$. The exiting transitions are $[x] \stackrel{b_{i}}{\longrightarrow}\left[x b_{i}\right]$ and $[x] \stackrel{\bar{b}_{i}}{\longrightarrow}\left[x \bar{b}_{i}\right]$, and we must show that $x b_{i} \not \equiv x \bar{b}_{i}$. But if $x b_{i} \equiv x \bar{b}_{i}$, then by Lemma 3.7 we would have $x \equiv x b_{i}$, which would contradict the assumption that $|\operatorname{last}(x)|=\operatorname{level}([x])$.

Lemma 3.9 For all $x \in$ PGS, $\sigma_{N}([x], \varepsilon)=[x]$.

Proof. We wish to show that $\operatorname{lcp}_{\alpha} \sigma_{N}([x], \alpha)=[x]$. If $[x]$ is an action state, then for all atoms $\alpha, \sigma_{N}([x], \alpha)=[x]$, and we are done. If $[x]$ is a test state, then its two successors are distinct by Lemma 3.8. We can pick atoms $\alpha$ and $\beta$ with opposite values for the $b \in \mathrm{B}$ tested at $[x]$, so the longest common prefix of $\sigma_{N}([x], \alpha)$ and $\sigma_{N}([x], \beta)$ is $[x]$. Thus $\sigma_{N}([x], \varepsilon)=\operatorname{lcp}_{\alpha} \sigma_{N}([x], \alpha)=[x]$.

Lemma 3.10 For all $x, y \in \mathbf{P G S}$, $\operatorname{last}\left(\sigma_{N}([x], y)\right)=[x \triangleleft y]$. In particular, $\operatorname{last}\left(\sigma_{N}([\varepsilon], x)\right)=[x]$.

Proof. Assume without loss of generality that $|\operatorname{last}(x)|=\operatorname{level}([x])$. First we show 
the result for $y=\omega$, a prefix of an atom. If $|\omega| \leq \operatorname{level}([x])$, then

$$
\begin{aligned}
\sigma_{N}([x], \omega) & =\underset{\alpha}{\operatorname{lcp}} \sigma_{N}([x], \omega \triangleleft \alpha) \\
& =\underset{\alpha}{\operatorname{lcp}} \sigma_{N}([x], \alpha) \\
& =\sigma_{N}([x], \varepsilon) \\
& =[x] \quad \text { by Lemma } 3.9 \\
& =[x \triangleleft \omega] .
\end{aligned}
$$

If $|\omega|>\operatorname{level}([x])$, let $i=\operatorname{level}([x])+1 \leq|\omega|$ and let $c \in\left\{b_{i}, \bar{b}_{i}\right\}$ such that $\omega \leq c$. For all atoms $\alpha$,

$$
\sigma_{N}([x], \omega \triangleleft \alpha)=([x] \stackrel{c}{\longrightarrow}[x c]) \cdot \sigma_{N}([x c], \omega \triangleleft \alpha),
$$

thus

$$
\begin{aligned}
\sigma_{N}([x], \omega) & =\underset{\alpha}{\operatorname{lcp}} \sigma_{N}([x], \omega \triangleleft \alpha) \\
& =([x] \stackrel{c}{\longrightarrow}[x c]) \cdot \underset{\alpha}{\operatorname{lcp}} \sigma_{N}([x c], \omega \triangleleft \alpha) \\
& =([x] \stackrel{c}{\longrightarrow}[x c]) \cdot \sigma_{N}([x c], \omega),
\end{aligned}
$$

therefore

$$
\begin{aligned}
\operatorname{last}\left(\sigma_{N}([x], \omega)\right) & =\operatorname{last}\left(([x] \stackrel{c}{\longrightarrow}[x c]) \cdot \sigma_{N}([x c], \omega)\right) \\
& =\operatorname{last}\left(\sigma_{N}([x c], \omega)\right) \\
& =[x c \triangleleft \omega] \quad \text { by the induction hypothesis } \\
& =[x \triangleleft \omega] .
\end{aligned}
$$

Finally, for $y p \omega \in \mathbf{P G S}$ where $y \in \mathbf{G S}, p \in \mathbf{P}$, and $\omega$ a prefix of an atom, by the induction hypothesis we have last $\left(\sigma_{N}([x], y)\right)=[x \triangleleft y]$. Then

$$
\begin{aligned}
& \sigma_{N}([x], y p \omega) \\
& \quad=\operatorname{lcp} \sigma_{N}([x], y p \omega \triangleleft \alpha) \\
& =\underset{\alpha}{\operatorname{lcp}}\left(\sigma_{N}([x], y) \cdot([x \triangleleft y] \stackrel{p}{\longrightarrow}[x \triangleleft y p]) \cdot \sigma_{N}([x \triangleleft y p], \omega \triangleleft \alpha)\right) \\
& =\sigma_{N}([x], y) \cdot([x \triangleleft y] \stackrel{p}{\longrightarrow}[x \triangleleft y p]) \cdot \operatorname{lc}_{\alpha} \sigma_{N}([x \triangleleft y p], \omega \triangleleft \alpha) \\
& =\sigma_{N}([x], y) \cdot([x \triangleleft y] \stackrel{p}{\longrightarrow}[x \triangleleft y p]) \cdot \sigma_{N}([x \triangleleft y p], \omega),
\end{aligned}
$$

thus

$$
\begin{aligned}
\operatorname{last} & \left(\sigma_{N}([x], y p \omega)\right) \\
= & \operatorname{last}\left(\sigma_{N}([x], y) \cdot([x \triangleleft y] \stackrel{p}{\longrightarrow}[x \triangleleft y p]) \cdot \sigma_{N}([x \triangleleft y p], \omega)\right) \\
= & \operatorname{last}\left(\sigma_{N}([x \triangleleft y p], \omega)\right) \\
= & {[(x \triangleleft y p) \triangleleft \omega] \quad \text { by the result for prefixes of atoms proved above } } \\
= & {[x \triangleleft y p \omega] . }
\end{aligned}
$$


Theorem 3.11 Up to isomorphism, the automaton $N$ constructed above is the unique minimal deterministic ordered AGS for A. Thus there are finitely many $\equiv$-classes iff $A$ is regular.

Proof. To show that $G(N)=A$, for any $x \in \mathbf{G S}, x \in G(N)$ iff $\operatorname{last}\left(\sigma_{N}([\varepsilon], x)\right)$ is a final state of $N$. By Lemmas 3.6 and 3.10, this occurs iff $x \in A$.

For any other deterministic ordered AGS $M$ for $A$, there is a surjective structurepreserving map from the accessible states of $M$ to the states of $N$. For $x, y \in \mathbf{P G S}$, define $x \sim y$ if last $\left(\sigma_{M}(s, x)\right)=\operatorname{last}\left(\sigma_{M}(s, y)\right)$, where $s$ is the start state of $M$. There is a one-to-one correspondence between the accessible states of $M$ and the $\sim$-equivalence classes. Moreover, if $x \sim y$, then $x \triangleleft z \in A$ iff $y \triangleleft z \in A$ for any $z$, therefore $x \equiv y$. Thus $\sim$ refines $\equiv$. The desired map is $\operatorname{last}\left(\sigma_{M}(s, x)\right) \mapsto[x]=\operatorname{last}\left(\sigma_{N}([\varepsilon], x)\right)$

\subsubsection{Unrestricted Tests}

If any test in $\mathcal{B}$ is allowed as an input symbol, we can adapt the construction of the previous section to give unique minimal deterministic automata.

Define $\mathbf{P G S}^{i}$ to be the set of prefixes $x$ of guarded strings such that $|\operatorname{last}(x)|=i$. Then $\mathbf{P G S}{ }^{0}=\{x p \mid x \in \mathbf{G S}, p \in \mathrm{P}\} \cup\{\varepsilon\}$ and $\mathbf{P G S} \mathbf{S}^{k}=\mathbf{G S}$. We define $\triangleleft$ and $\equiv$ as in Section 3.3.1 and take $\left\{[x] \mid x \in \mathbf{P G S}^{0} \cup \mathbf{P G S}^{k}\right\}$ as states of our automaton. The equivalence classes $[x]$ for $x \in \mathbf{G S}$ are the action states. The remaining states are test states. The transitions are

$$
[y] \stackrel{\alpha}{\longrightarrow}[y \alpha] \quad[x] \stackrel{p}{\longrightarrow}[x p]
$$

for $\alpha \in \mathcal{A}_{\mathrm{B}}, p \in \mathbf{P}, x \in \mathbf{G S}$, and $y \in \mathbf{P G S}^{0}$ such that $y \not \equiv z$ for any $z \in \mathbf{G S}$. The start state is $[\varepsilon]$ and the final states are $\{[x] \mid x \in A\}$. A direct adaptation of the arguments of Section 3.3.1 shows that there are finitely many $\equiv$-classes iff $A$ is regular, and that this construction gives the minimal deterministic AGS for $A$ over the alphabet $\mathrm{P} \cup \mathcal{B}$.

\section{Applications}

\subsection{The Complexity of System S}

System S, introduced in [10], is a Gentzen-style sequent calculus for partial correctness that subsumes propositional Hoare Logic. It was shown in [10] that the system is sound and complete over relational and trace-based models. 
The syntax of system $\mathrm{S}$ is given in the following grammar. Here we revert to the notation of [10], in which + is written as $\oplus$ and $\cdot$ as $\otimes$. Also, the positive iteration operator ${ }^{+}$is taken as primitive, and ${ }^{*}$ is defined by $p^{*} \stackrel{\text { def }}{=} \mathbf{1} \oplus p^{+}$. Note that there are two kinds of propositions, tests and formulas.

$$
\begin{array}{lll}
\text { tests } & b, c, d \ldots & b::=\langle\text { atomic tests }\rangle|\mathbf{0}| b \rightarrow c \\
\text { programs } & p, q, r, \ldots & p::=\langle\text { atomic actions }\rangle|b| p \oplus q|p \otimes q| p^{+} \\
\text {formulas } & \varphi, \psi, \ldots & \varphi::=b \mid p \rightarrow \varphi \\
\text { environments } & \Gamma, \Delta, \ldots & \Gamma::=\varepsilon|\Gamma, p| \Gamma, \varphi \\
\text { sequents } & \Gamma \vdash \varphi
\end{array}
$$

We abbreviate $b \rightarrow \mathbf{0}$ by $\bar{b}, \overline{\mathbf{0}}$ by $\mathbf{1}$, and $p \otimes q$ by $p q$.

A formula is either a test or an expression $p \rightarrow \varphi$, read "after $p, \varphi$," where $p$ is a program and $\varphi$ is a formula. Intuitively, the meaning is similar to the modal construct $[p] \varphi$ of Dynamic Logic (DL) (see [3]). The operator $\rightarrow$ associates to the right. The empty environment is denoted $\varepsilon$. Intuitively, an environment describes a previous computation that has led to the current state. Sequents are of the form $\Gamma \vdash \varphi$, where $\Gamma$ is an environment and $\varphi$ is a formula. We write $\vdash \varphi$ for $\varepsilon \vdash \varphi$. Intuitively, the meaning of $\Gamma \vdash \varphi$ is similar to the DL assertion $[\Gamma] \varphi$, where we think of the environment $\Gamma=\ldots, p, \ldots, \psi, \ldots$ as the rich-test program $\cdots ; p ; \cdots ; \psi ? ; \cdots$ of DL.

It is shown in [10] how to encode propositional Hoare Logic (PHL). It follows from the completeness theorem of [10] that all relationally valid Hoare rules are derivable; this is false for PHL [7, 9].

Programs and tests are interpreted over Kripke frames $K$ as described in Section 2.2. Additionally, we interpret formulas, environments, and sequents as follows:

$$
\begin{aligned}
& \left.\left.[[p \rightarrow \varphi]]_{K} \stackrel{\text { def }}{=}\{s \mid \forall \tau \operatorname{first}(\tau)=s \text { and } \tau \in \llbracket p]\right]_{K} \Rightarrow \operatorname{last}(\tau) \in[[\varphi]]_{K}\right\} \\
& {[[\varepsilon]]_{K} \stackrel{\text { def }}{=} K} \\
& {\left[[\Gamma, \Delta \rrbracket]_{K} \stackrel{\text { def }}{=}[[\Gamma]]_{K} \circ[[\Delta]]_{K}\right. \text {. }}
\end{aligned}
$$

The sequent $\Gamma \vdash \varphi$ is valid in the trace model $K$ if for all traces $\sigma \in \llbracket \Gamma]_{K}$, last $(\sigma) \in$ $[[\varphi]]_{K}$; equivalently, if $\left[[\Gamma]_{K} \subseteq[[\Gamma, \varphi]]_{K}\right.$.

The rules of S are given in Fig. 2. It was shown in [10] that this system is sound and complete over trace models; that is, the sequent $\Gamma \vdash \varphi$ is valid in all trace models iff it is derivable in this deductive system.

A rule is admissible if for any substitution instance for which the premises are provable, the conclusion is also provable. It was shown in [10] that the rule and sequent

$$
(\mathbf{E R} \rightarrow) \quad \frac{\Gamma \vdash p \rightarrow \varphi}{\Gamma, p \vdash \varphi} \quad \text { (ident) } \varphi \vdash \varphi
$$




\section{Axiom ( $b$ is a test):}

$b \vdash b$

Test-cut Rule ( $b$ is a test):

$$
\text { (test-cut) } \frac{\Gamma, b, \Delta \vdash \varphi \quad \Gamma, \bar{b}, \Delta \vdash \varphi}{\Gamma, \Delta \vdash \varphi}
$$

\section{Introduction Rules:}

$$
\begin{array}{ll}
\text { (I } \otimes) & \frac{\Gamma, p, q, \Delta \vdash \varphi}{\Gamma, p \otimes q, \Delta \vdash \varphi} \\
\text { (I } \oplus) & \frac{\Gamma, p, \Delta \vdash \varphi \quad \Gamma, q, \Delta \vdash \varphi}{\Gamma, p \oplus q, \Delta \vdash \varphi} \\
\text { (I 0) } & \Gamma, \mathbf{0}, \Delta \vdash \varphi \\
\text { (I } \left.^{+}\right) & \frac{\psi, p \vdash \varphi \quad \psi, p \vdash \psi}{\psi, p^{+} \vdash \varphi}
\end{array}
$$

\section{Structural Rules:}

$$
\begin{array}{ll}
(\mathbf{W} \psi) & \frac{\Gamma, \Delta \vdash \varphi}{\Gamma, \psi, \Delta \vdash \varphi} \\
(\mathbf{W} p) & \frac{\Gamma \vdash \varphi}{p, \Gamma \vdash \varphi} \\
\left(\mathbf{C C}^{+}\right) & \frac{\Gamma, p^{+}, \Delta \vdash \varphi}{\Gamma, p^{+}, p^{+}, \Delta \vdash \varphi}
\end{array}
$$

\section{Arrow Rules:}

$$
\begin{array}{ll}
(\mathbf{R} \rightarrow) & \frac{\Gamma, p \vdash \varphi}{\Gamma \vdash p \rightarrow \varphi} \\
(\mathbf{I} \rightarrow) & \frac{\Gamma, p, \psi, \Delta \vdash \varphi}{\Gamma, p \rightarrow \psi, p, \Delta \vdash \varphi}
\end{array}
$$

Elimination Rules:

$$
\begin{array}{ll}
(\mathbf{E} \otimes) & \frac{\Gamma, p \otimes q, \Delta \vdash \varphi}{\Gamma, p, q, \Delta \vdash \varphi} \\
(\mathbf{E} 1 \oplus) & \frac{\Gamma, p \oplus q, \Delta \vdash \varphi}{\Gamma, p, \Delta \vdash \varphi} \\
(\mathbf{E 2} \oplus) & \frac{\Gamma, p \oplus q, \Delta \vdash \varphi}{\Gamma, q, \Delta \vdash \varphi} \\
\left(\mathbf{E}^{+}\right) & \frac{\Gamma, p^{+}, \Delta \vdash \varphi}{\Gamma, p, \Delta \vdash \varphi}
\end{array}
$$

\section{Cut Rule:}

$$
\text { (cut) } \frac{\Gamma \vdash \psi \quad \Gamma, \psi, \Delta \vdash \varphi}{\Gamma, \Delta \vdash \varphi}
$$

Figure 2: Rules of System S [10] 
are admissible.

Lemma 4.1 The operator $\otimes$ and rules $(\mathbf{I} \otimes)$ and $(\mathbf{E} \otimes)$ can be extended to pairs of formulas in the following sense: there exists a map $\varphi, \psi \mapsto \varphi \otimes \psi$ such that the rules

$$
\frac{\Gamma, \varphi, \psi, \Delta \vdash \rho}{\Gamma, \varphi \otimes \psi, \Delta \vdash \rho} \quad \frac{\Gamma, \varphi \otimes \psi, \Delta \vdash \rho}{\Gamma, \varphi, \psi, \Delta \vdash \rho}
$$

are admissible. We use $(\mathbf{I} \otimes)$ and $(\mathbf{E} \otimes)$, respectively, to refer to these extended rules as well.

Proof. If $\varphi=p_{1} \rightarrow \cdots \rightarrow p_{m} \rightarrow b$ and $\psi=q_{1} \rightarrow \cdots \rightarrow q_{n} \rightarrow c$, define

$$
\varphi \otimes \psi \stackrel{\text { def }}{=}\left(p_{1} \cdots p_{m} \bar{b} \oplus q_{1} \cdots q_{n} \bar{c}\right) \rightarrow \mathbf{0} .
$$

Using $(\mathbf{R} \rightarrow),(\mathbf{E R} \rightarrow)$, and $(\mathbf{I} \otimes)$ and $(\mathbf{E} \otimes)$ on programs, it can be shown that

$$
\begin{aligned}
p_{1} \rightarrow \cdots \rightarrow p_{m} \rightarrow b & \vdash \quad p_{1} \cdots p_{m} \bar{b} \rightarrow \mathbf{0} \\
p_{1} \cdots p_{m} \bar{b} \rightarrow \mathbf{0} & \vdash \quad p_{1} \rightarrow \cdots \rightarrow p_{m} \rightarrow b .
\end{aligned}
$$

We also have

$$
p \rightarrow \mathbf{0}, q \rightarrow \mathbf{0} \vdash p \oplus q \rightarrow \mathbf{0} \quad p \oplus q \rightarrow \mathbf{0} \vdash p \rightarrow \mathbf{0} \quad p \oplus q \rightarrow \mathbf{0} \vdash q \rightarrow \mathbf{0}
$$

by the following arguments:

$$
\begin{gathered}
\frac{p \rightarrow \mathbf{0} \vdash p \rightarrow \mathbf{0}}{p \rightarrow \mathbf{0}, q \rightarrow \mathbf{0}, p \vdash \mathbf{0}}\left(\mathbf{E R \rightarrow} \rightarrow,(\mathbf{W} \psi) \quad \frac{q \rightarrow \mathbf{0} \vdash q \rightarrow \mathbf{0}}{p \rightarrow \mathbf{0}, q \rightarrow \mathbf{0}, q \vdash \mathbf{0}}\right. \\
\frac{p \rightarrow \mathbf{0}, q \rightarrow \mathbf{0}, p \oplus q \vdash \mathbf{0}}{p \rightarrow \mathbf{0}, q \rightarrow \mathbf{0} \vdash p \oplus q \rightarrow \mathbf{0}}(\mathbf{R} \rightarrow) \\
(\mathbf{I} \oplus) \\
\frac{p \oplus q \rightarrow \mathbf{0} \vdash p \oplus q \rightarrow \mathbf{0}}{p \oplus q \rightarrow \mathbf{0}, p \oplus q \vdash \mathbf{0}}(\mathbf{E R} \rightarrow) \\
\frac{p \oplus q \rightarrow \mathbf{0}, p \vdash \mathbf{0}}{p \oplus q \rightarrow \mathbf{0} \vdash p \rightarrow \mathbf{0}}(\mathbf{R} \rightarrow) \quad \frac{p \oplus q \rightarrow \mathbf{0}, q \vdash \mathbf{0}}{p \oplus q \rightarrow \mathbf{0} \vdash q \rightarrow \mathbf{0}}(\mathbf{R} \rightarrow) \\
(\mathbf{E} 1 \oplus),(\mathbf{E} \mathbf{2} \oplus)
\end{gathered}
$$

It follows from (cut) that

$$
\varphi, \psi \vdash \varphi \otimes \psi \quad \varphi \otimes \psi \vdash \varphi \quad \varphi \otimes \psi \vdash \psi .
$$

The admissibility of the extended $(\mathbf{I} \otimes)$ and $(\mathbf{E} \otimes)$ then follows from (cut).

Lemma 4.2 Let $w=(\bigoplus \mathrm{P})^{*}$ be an expression denoting all guarded strings. The sequent

$$
q_{1} \rightarrow \mathbf{0}, p_{1}, q_{2} \rightarrow \mathbf{0}, p_{2}, \ldots, q_{n} \rightarrow \mathbf{0}, p_{n} \quad \vdash \quad \mathbf{0}
$$

is valid if and only if there do not exist guarded strings $x_{1}, \ldots, x_{n}$ such that $x_{1} \cdots x_{n}$ exists, $x_{i} \in G\left(p_{i}\right), 1 \leq i \leq n$, and $x_{i} x_{i+1} \cdots x_{n} \notin G\left(q_{i} w\right), 1 \leq i \leq n$. 
Proof. Suppose such $x_{1}, \ldots, x_{n}$ exist. Let $\Gamma$ be the environment on the left-hand side of (3). Construct a trace model $K$ consisting of a single linear trace $\sigma$ such that $\operatorname{gs}(\sigma)=x_{1} \cdots x_{n}$. The model is uniquely determined by this specification. Let $\sigma_{i}$, $1 \leq i \leq n$, be the unique subtraces of $\sigma$ such that gs $\left(\sigma_{i}\right)=x_{i}$ and $\sigma=\sigma_{1} \cdots \sigma_{n}$. By Lemma 2.1, $\sigma_{i} \in \llbracket p_{i} \rrbracket_{K}$. Since $x_{i} x_{i+1} \cdots x_{n} \notin G\left(q_{i} w\right)$, no prefix of $x_{i} x_{i+1} \cdots x_{n}$ is in $G\left(q_{i}\right)$, so by Lemma 2.1 no prefix of $\sigma_{i} \sigma_{i+1} \cdots \sigma_{n}$ is in $\left[\left[q_{i}\right]_{K}\right.$. Since these are the only traces in $K$ with initial state first $\left(\sigma_{i}\right)$, we have first $\left(\sigma_{i}\right) \in \llbracket q_{i} \rightarrow \mathbf{0} \rrbracket_{K}$, therefore $\sigma_{i} \in \llbracket\left[q_{i} \rightarrow \mathbf{0}, p_{i}\right]_{K}$. It follows that $\left.\sigma \in \llbracket \Gamma\right]_{K}$. Since $\llbracket \Gamma \rrbracket_{K}$ is nonempty, (3) is not valid.

Conversely, suppose (3) is not valid. Let $K$ be a trace model and $\sigma$ a trace in $K$ such that $\sigma \in \llbracket \Gamma \rrbracket_{K}$. There exist subtraces $\sigma_{i}$ in $K, 1 \leq i \leq n$, such that $\sigma=$ $\sigma_{1} \cdots \sigma_{n}$ and $\sigma_{i} \in \llbracket q_{i} \rightarrow \mathbf{0}, p_{i} \rrbracket_{K}$. Then $\left.\operatorname{first}\left(\sigma_{i}\right) \in \llbracket q_{i} \rightarrow \mathbf{0}\right]_{K}$, so no prefix of $\sigma_{i} \sigma_{i+1} \cdots \sigma_{n}$ is in $\llbracket q_{i} \rrbracket_{K}$, and $\sigma_{i} \in \llbracket p_{i} \rrbracket_{K}$. Let $x_{i}=\operatorname{gs}\left(\sigma_{i}\right)$. By Lemma 2.1, no prefix of $x_{i} x_{i+1} \cdots x_{n}$ is in $G\left(q_{i}\right)$, therefore $x_{i} x_{i+1} \cdots x_{n} \notin G\left(q_{i} w\right)$, and $x_{i} \in G\left(p_{i}\right)$, $1 \leq i \leq n$.

Theorem 4.3 The problem of deciding whether a given sequent of System $\mathrm{S}$ is valid is PSPACE-complete.

Proof. As observed in [10], the problem encodes the equivalence problem for regular expressions, a well-known PSPACE-complete problem [11], therefore is PSPACEhard. It thus remains to show that the problem is in PSPACE.

Suppose we are given a sequent of the form

$$
q_{1} \rightarrow \mathbf{0}, p_{1}, q_{2} \rightarrow \mathbf{0}, p_{2}, \ldots, q_{n} \rightarrow \mathbf{0}, p_{n} \vdash \mathbf{0} .
$$

Using the extended $(\mathbf{I} \otimes)$ and $(\mathbf{E} \otimes)$ of Lemma 4.1 along with $(\mathbf{E R} \rightarrow)$ and $(\mathbf{R} \rightarrow)$, we can transform any given sequent to one of this form with no significant increase in size, so the assumption is without loss of generality.

Now build nondeterministic automata $M_{i}$ from the $p_{i}$ and $N_{i}$ from the $q_{i} w$ as in Theorem 3.1, where $w=(\bigoplus \mathrm{P})^{*}$ is an expression representing all guarded strings. Our PSPACE algorithm will guess guarded strings $x_{1}, \ldots, x_{n}$ symbol by symbol in that order, scanning the automata to check the positive and negative conditions of Lemma 4.2. We must check that the automata $M_{i}$ accept the $x_{i}$ and the automata $N_{i}$ reject $x_{i} x_{i+1} \cdots x_{n}$. This is done by simulating the subset construction of Section 3.2 with pebbles occupying the states of the $M_{i}$ and $N_{i}$. After every guessed atomic action or atom, the pebbles are moved according to the transitions of the deterministic automata constructed in Section 3.2. Guessing an atom amounts to guessing a truth assignment to $B$; then to determine whether a test transition is enabled, we just evaluate the label on that truth assignment. We also guess the boundaries between the $x_{i}$ and $x_{i+1}$ and make sure that last $\left(x_{i}\right)=\operatorname{first}\left(x_{i+1}\right)$. The entire simulation can be done in PSPACE, since by Theorem 3.1, the automata $M_{i}$ and $N_{i}$ are linear in the size of the expressions $p_{i}$ and $q_{i} w$, respectively, and the simulation need only maintain pebble configurations 
on each nondeterministic automaton. It does not matter how long the strings $x_{i}$ are; the simulation continues to guess symbols until it succeeds (or not). This gives a nondeterministic PSPACE algorithm, which can be made deterministic using Savitch's theorem.

\subsection{Boolean Decision Diagrams}

We refer the reader to Andersen's lecture notes [1] for an introduction to BDDs. A BDD is ordered (OBDD) if the order of the tests along any path is consistent with a given linear order on B. An OBDD is reduced (ROBDD) if (i) no two nodes that test the same Boolean variable have the same true successors and the same false successors, and (ii) the true and false successors of any node are distinct. The Canonicity Lemma ([1, p. 13]) says that any Boolean function has a unique ROBDD for a given linear order on B. The next theorem shows that the Canonicity Lemma is essentially Theorem 3.11 in the special case $P=\varnothing$.

Theorem 4.4 Let $\mathrm{P}=\varnothing$. For any $A \subseteq \mathcal{A}_{\mathrm{B}}$, the minimal ordered AGS for $A$ for $a$ given order on $\mathrm{B}$ constructed in Section 3.3.1 is the canonical ROBDD for $\bigvee A$ with respect to that order.

Proof. The AGS is apparently an OBDD for $\bigvee A$. It therefore remains to check conditions (i) and (ii).

For condition (i), suppose level $([x])=\operatorname{level}([y])=i-1$. Assume without loss of generality that $|\operatorname{last}(x)|=|\operatorname{last}(y)|=i-1$. If $\left[x b_{i}\right]=\left[y b_{i}\right]$ and $\left[x \bar{b}_{i}\right]=\left[y \bar{b}_{i}\right]$, then $x b_{i} \equiv y b_{i}$ and $x \bar{b}_{i} \equiv y \bar{b}_{i}$. Let $z \in \mathbf{G S}$ be arbitrary. If $b_{i}$ occurs positively in first $(z)$, then $x \triangleleft z \in A$ iff $x b_{i} \triangleleft z \in A$ iff $y b_{i} \triangleleft z \in A$ iff $y \triangleleft z \in A$. Similarly, if $b_{i}$ occurs negatively in $\operatorname{first}(z)$, then $x \triangleleft z \in A$ iff $x \bar{b}_{i} \triangleleft z \in A$ iff $y \bar{b}_{i} \triangleleft z \in A$ iff $y \triangleleft z \in A$. Thus $x \equiv y$ and $[x]=[y]$.

Condition (ii) is just Lemma 3.8 .

\section{Acknowledgements}

I am indebted to Jerzy Tiuryn for many valuable ideas and engaging discussions. This work grew out of joint work with him [9, 10]. I also thank the anonymous referee for valuable suggestions for improving the presentation. This work was supported in part by NSF grant CCR-0105586 and by ONR Grant N00014-01-1-0968. The views and conclusions contained herein are those of the author and should not be interpreted as necessarily representing the official policies or endorsements, either expressed or implied, of these organizations or the US Government. 


\section{References}

[1] Henrik Reif Andersen. An introduction to binary decision diagrams. Lecture notes, Department of Information Technology, Technical University of Denmark, Copenhagen. http://www.itu.dk/people/hra/notes-index.html, April 1998.

[2] Ernie Cohen, Dexter Kozen, and Frederick Smith. The complexity of Kleene algebra with tests. Technical Report 96-1598, Computer Science Department, Cornell University, July 1996.

[3] David Harel, Dexter Kozen, and Jerzy Tiuryn. Dynamic Logic. MIT Press, Cambridge, MA, 2000.

[4] Donald M. Kaplan. Regular expressions and the equivalence of programs. J. Comput. Syst. Sci., 3:361-386, 1969.

[5] Dexter Kozen. Automata and Computability. Springer-Verlag, New York, 1997.

[6] Dexter Kozen. Kleene algebra with tests. Transactions on Programming Languages and Systems, 19(3):427-443, May 1997.

[7] Dexter Kozen. On Hoare logic and Kleene algebra with tests. Trans. Computational Logic, 1(1):60-76, July 2000.

[8] Dexter Kozen and Frederick Smith. Kleene algebra with tests: Completeness and decidability. In D. van Dalen and M. Bezem, editors, Proc. 10th Int. Workshop Computer Science Logic (CSL'96), volume 1258 of Lecture Notes in Computer Science, pages 244-259, Utrecht, The Netherlands, September 1996. Springer-Verlag.

[9] Dexter Kozen and Jerzy Tiuryn. On the completeness of propositional Hoare logic. Information Sciences, 139(3-4):187-195, 2001.

[10] Dexter Kozen and Jerzy Tiuryn. Substructural logic and partial correctness. Trans. Computational Logic, 4(3), July 2003.

[11] L. J. Stockmeyer and A. R. Meyer. Word problems requiring exponential time. In Proc. 5th Symp. Theory of Computing, pages 1-9, New York, 1973. ACM. 